IOS Press

\title{
Variations in upper limb and trunk muscles activation during isometric exercises with or without exertion of control
}

\author{
Koji Murofushi ${ }^{\mathrm{a}, *}$, Daisuke Yamaguchi ${ }^{\mathrm{a}}$, Kenji Hirohata ${ }^{\mathrm{b}}$, Hidetaka Furuya ${ }^{\mathrm{c}}$ and Sho Mitomo ${ }^{\mathrm{b}}$ \\ ${ }^{a}$ Sports Science Center, Tokyo Medical and Dental University (TMDU), Tokyo, Japan \\ ${ }^{\mathrm{b}}$ Clinical Center for Sports Medicine and Sports Dentistry, Tokyo Medical and Dental University, Tokyo, Japan \\ ${ }^{\mathrm{c}}$ Department of Rehabilitation, Sonoda Third Hospital/Tokyo Medical Institute Tokyo Spine Center, Tokyo, Japan
}

Received 10 October 2021

Accepted 6 January 2022

\begin{abstract}
.
BACKGROUND: Muscle activation during isometric contraction can be changed using an external-focus instruction method. OBJECTIVE: To explore and compare the activity of eight upper limb and trunk muscles during isometric chest squeeze exercises, performed in the same posture with and without the exertion of control to avoid crushing an object.

METHODS: Muscle activation by an isometric chest squeeze with $45^{\circ}$ shoulder abduction and forearm pronation was examined. Isometric chest squeeze exercises were performed while holding objects with maximum pressure, and with exerted control to avoid crushing the object Muscle activation was measured by surface electromyography.

RESULTS: During the exercises performed while exerting control to avoid crushing the object the lower trapezius $(p<0.001)$ and latissimus dorsi $(p=0.03$ ) showed significantly higher activity. During the exercises performed without control, the upper trapezius, serratus anterior, and pectoralis major showed significantly higher activity $(p=0.016, p<0.001, p<0.001$, respectively).

CONCLUSIONS: This novel isometric exercise can increase the scapular muscles in the intermediate range of the glenohumeral joint. Therefore, it may assist in improving sport performance, in preventing injuries and in rehabilitation following musculoskeletal injuries.
\end{abstract}

Keywords: Isometric contraction, trapezius muscle, shoulder, instruction, exercise therapy

\section{Introduction}

Instructions to athletes, patients, and clients include information on the muscles involved in various activities while teaching how to perform exercises effectively. Muscle activity can change with different instruction methods [1]. Therefore, it is important to know what individuals should focus their attention on throughout

\footnotetext{
*Corresponding author: Koji Murofushi, Special Appointed Professor, Koji Murofushi Sports Science Center, Tokyo Medical and Dental University, zip code 113-0034, 1-5-45 Ushima, Bunkyo-ku, Tokyo, Japan. E-mail: kojimuro.ssc@tmd.ac.jp.
}

the instruction. The focus of attention can be classified as either internal or external. Internal focus is when individuals focus on and consciously control their movements. On the other hand, external focus is when individuals direct their attention to the movement's effect.

Internal focus, such as focusing on specific muscles or body parts, has a measurable effect on changing muscle activity, even during various activities with no change in posture [1-3]. Meanwhile, external-focus instructions can prompt individuals to focus on the movements' intended effects on the environment or on an external object related to the performed task. The use of external focus has been shown to produce a variety of 
effects, such as improving force production during an arm curl [4] and increasing the standing long jump distance [5]. Wulf et al. indicated that the performance of externally-focused jumps was significantly better than that of internally focused jumps, with generally lower electromyography (EMG) activity [6].

Isometric exercises are widely used in rehabilitation for patients, in preparatory exercises for athletes, and for the general public to improve their health. Isometric exercises are characterized by the fact that they do not involve joint movement, thereby may cause under comparable conditions, less stress on the joints. Thus, compared to their dynamic counterparts, isometric exercises are easier to perform even when there are difficulties in moving the joints, such as immediately after an injury or a surgery for musculoskeletal injury or disease. There are several ways to perform an exercise to achieve an isometric muscle contraction [7-10]. Regardless of the unweighted or weighted isometric exercise, maintaining the muscle length and joint angle during muscle activation is necessary. For example, pushing the hands together or against a wall can help consciously fix and hold a certain posture, which helps maintain the muscle length and joint angle at the same level. Previous reports have shown that the amount of muscle activity differs depending on the verbal instruction $[11,12]$. In these reports, several types of instructions with external focus were used, such as focusing on the movement of the barbell or the bar. These reports analyzed muscle activity during an isokinetic exercise, and it is unclear how verbal instructions using external focus affect muscle activity during an isometric exercise.

There is a possibility that the verbal instructions that evoke external focus to control an object without crushing it may play an important role in differentially activating the muscles that stabilize the upper body. If changes in muscle activity due to differences in instructions can be identified, useful information may be obtained for exercise and rehabilitation programs and in particular, those targeting shoulder and scapula stabilization programs during periods of limited joint movement, occurring in the early phase following an injury or a surgery for upper limb musculoskeletal diseases. Furthermore, to the best of our knowledge, such an exercise has not been described.

Therefore, the purpose of this study was to confirm the alteration of muscle activity during isometric chest squeeze exercises, performed while exerting control to avoid crushing an object whilst maintaining a static position holding a soft paper balloon. We also assessed muscle activity during isometric chest squeeze exercises
Table 1

Participants' characteristics

\begin{tabular}{ll}
\hline \multicolumn{1}{c}{ Parameters } & \multicolumn{1}{c}{ Values } \\
\hline Number of participants & 24 \\
Age (years) & $31(19-49)$ \\
Height $(\mathrm{cm})$ & $175(166-189)$ \\
Weight $(\mathrm{kg})$ & $75(58-107)$ \\
BMI $\left(\mathrm{kg} / \mathrm{m}^{2}\right)$ & $24.4(20.3-31.7)$ \\
\hline
\end{tabular}

BMI, body mass index; $n$, number.

performed without exerting control to avoid crushing an object using a hard-plastic cylinder. We hypothesized that isometric exercises performed while exerting control to avoid crushing an object would increase muscles activity to keep the upper limb elevated compared to the traditional isometric method.

\section{Methods}

\subsection{Participants}

Twenty-four healthy male adults aged 19-49 years, participated in this study (Table 1). An a priori sample size calculation was conducted with $\mathrm{G}^{*}$ power software 3.1.9.4 (Franz, Universitat Kiel, Germany). Based on a pilot study which compared muscle activity using the paper balloon method (PBM) and the conventional isometric method (CIM), the minimum number was 20 participants (effect size $=0.93$, alpha $=0.05$, power $=$ 0.80 , two-tailed). All participants were physically active and declared three practice sessions per week as part of a regular exercise routine, but had no experience with an isometric chest squeeze in their exercise routines. Before the experiment began, all participants who had a serious injury within the last 3 months and experienced pain on the day of examination were excluded. The participants were instructed to stop when they felt any pain during any part of the test. None of the participants discontinued due to injury or pain throughout the study.

\subsection{Study design}

This was an experimental study that used a withinparticipant repeated-measures design. Muscle activity was the dependent variable, and the type of exercise was the independent variable. The study was approved by the Research Ethics Committee of the participating institution (approval number: M2019-295) on 4 March 2020 and followed the principles of the Declaration of Helsinki (52nd WMA General Assembly Edinburgh, Scotland, October 2000) for Medical Research Involving Human Subjects. All participants provided written informed consent for participation in this study before engaging in the trials. 


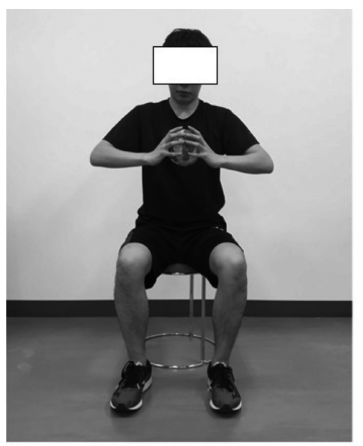

(a)

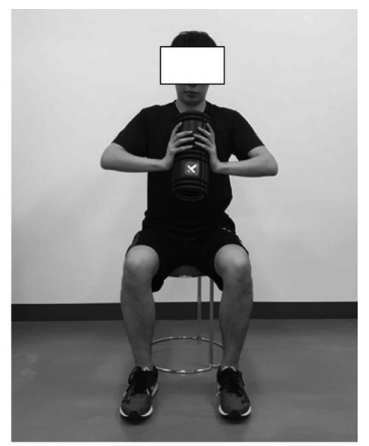

(b)
Fig. 1. a) Paper balloon setup position. b) Hard-plastic cylinder setup position.

\subsection{Procedures}

Two types of materials, soft paper balloon and hard plastic cylinder, were chosen in the exercises, and wireless surface EMG was used to compare changes in muscle activation and its variability in the same participants and in the same session. For the exercise, we selected the isometric chest squeeze as it requires holding the materials on both sides and maintaining the same body position. Muscle activation when performing novel isometric method using a soft paper balloon with control exerted to avoid crushing the object was compared with that performed without exerting control using a hard-plastic cylinder in the same posture. We asked the participants to maintain their body position and hold the object while exerting control to avoid crushing the soft paper balloon (Fig. 1a), and defined this activity as the paper balloon method (PBM). For the regular isometric exercise, we selected a hard-plastic cylinder (Fig. 1b) and asked the participants to perform the exercise without exerting control to avoid crushing the cylinder, and defined this activity as the conventional isometric method (CIM). Both objects had the same diameter and width so that the hand position would be the same between both exercises, and the participants pushed against the object in the same posture.

\subsection{Paper balloon}

For the PBM, we selected a soft paper balloon, or Kamifusen (紙風船), a traditional Japanese toy balloon with a small hole made from rice paper. Despite the hole, the paper balloon remains inflated due to its ability to maintain proportional air pressure between the inside and the outside [13]. During the chest squeezing exercise, a crumpling paper sound signaled that the balloon was crushed (configuration of the paper balloon: diameter, $14 \mathrm{~cm}$; weight, $5 \mathrm{~g}$ ).

\subsection{Hard-plastic cylinder}

For the CIM, we selected a hard-plastic material covered by polyurethane in a cylindrical shape, which can withstand pressure during conditions of maximal effort in the trials (configuration of the GRID ${ }^{\circledR}$ Foam Roller (Durham NC, USA): height, $33 \mathrm{~cm}$; diameter, $14 \mathrm{~cm}$; weight, $650 \mathrm{~g}$; weight limit, $226.7 \mathrm{~kg}$ ).

\subsection{Trials}

All the measurements were performed in one day, one session. The participants performed four trials, with two trials each for PBM and CIM. The duration of the trials was determined based on the preliminary study and the participants' possible fatigue due to the maximum muscle exertion during the exercise task. The participants were given 90-s intervals between each performance for rest [14]. The participants were also instructed to perform the PBM and CIM under the same conditions. Before starting the task, the posture was always visually evaluated by the examiner. Furthermore, the posture was continuously controlled during the task. The participants were instructed to sit on the chair in a natural straight position with the knees and hips flexed at $90^{\circ}$ and both hands maintaining the paper balloon or the hard cylinder in front of their chest, at the height of the clavicles. The shoulders were placed at $45^{\circ} \mathrm{ab}-$ duction, and the forearms were pronated (Fig. 1a and b). The participants were asked to remain in the same position for the chest squeeze. Verbal instructions were provided to each participant before the trials to ensure proper squeezing of the equipment. In both the PBM and CIM, the participants were instructed to squeeze the object for $10 \mathrm{~s}$ with maximum effort using both hands. Furthermore, in the PBM, the participants were instructed to "push with maximum force, being careful not to crush the paper balloon." In the CIM, because of the hard cylinder's stiffness, the muscle length and joint angle were maintained. In the PBM, the muscle length and joint angle were held by maintaining the shape of the object and position of the hands during maximal squeeze effort (Table 2). Participants immediately realized when the paper balloon was crushed by the sound of the paper crumpling, thus knowing when the hands were not maintaining position. We did not give any instruction to focus on a specific body part or muscle group (internal-focus instruction). The instructions were designed such that participants would focus on an external object related to the performed task (externalfocus instruction) during the exercise. For each PBM 
Table 2

Standardized instructions for isometric exercises

\begin{tabular}{lll}
\hline \multicolumn{1}{c}{ Exercise } & \multicolumn{1}{c}{ Instruction } & \multicolumn{1}{c}{ Set up } \\
\hline Paper balloon method & $\begin{array}{l}\text { With the start call, squeeze the paper } \\
\text { balloon as hard as you can without } \\
\text { crushing it. Try to keep the shape of the } \\
\text { paper balloon. Do not change the }\end{array}$ & $\begin{array}{l}\text { Sit naturally straight on the chair with the } \\
\text { knees and hips flexed at about } 90^{\circ}, \text { and, } \\
\text { with both hands, bring the paper balloon } \\
\text { posture. }\end{array}$ \\
& $\begin{array}{l}\text { or cylinder in front of the chest, between } \\
\text { the clavicle bones to the setting posture. }\end{array}$ \\
Conventional isometric method & $\begin{array}{l}\text { With the start call, squeeze the cylinder } \\
\text { as hard as you can. Do not change the } \\
\text { posture. }\end{array}$ \\
&
\end{tabular}

Table 3

Placement of the EMG electrodes

\begin{tabular}{ll}
\hline \multicolumn{1}{c}{ Muscle } & \multicolumn{1}{c}{ Electrode placement location } \\
\hline Upper trapezius & One-half the distance between the C7 spinous process and the lateral tip of the acromion \\
Lower trapezius & Two-thirds of the way up the line from the scapular spine root to the eighth thoracic vertebra \\
Serratus anterior & Vertically along the midaxillary line at rib levels 6 through 8 \\
Latissimus dorsi & $4 \mathrm{~cm}$ below the lower corner of the scapula \\
Clavicular part of pectoralis major & Median muscle belly along the pectoralis major clavicular fibers \\
External oblique & Midpoint between the superior anterior iliac spine and the lower end of the ribs \\
Internal oblique & Approximately $2 \mathrm{~cm}$ medial and inferior to the ASIS \\
Rectus abdominis & Right aspects of the umbilicus and oriented parallel with the muscle fibers \\
\hline
\end{tabular}

EMG, electromyography; ASIS, anterior superior iliac spine.
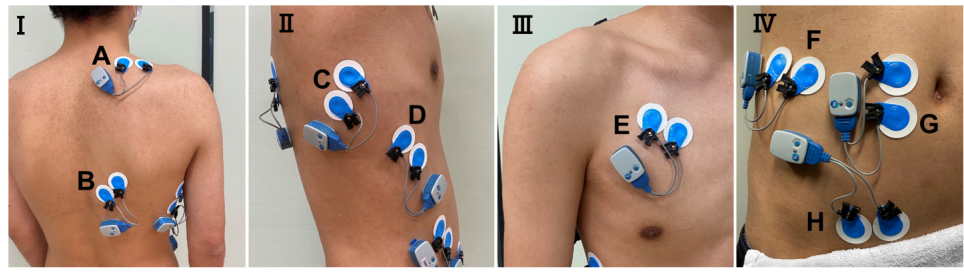

Fig. 2. Electrode application site for electromyography. I: Posterior view, II: lateral view, III: frontal view of the chest, IV: frontal view of the abdomen. A: Upper Trapezius, B: Lower Trapezius, C: Latissimus Dorsi, D: Serratus Anterior, E: Clavicular part of Pectoralis Major, F: External Oblique, G: Rectus Abdominis, H: Internal Oblique.

session, we ensured that the paper balloon was properly inflated. All participants had no experience with the PBM exercise, but each watched an instructional video before coming to the laboratory. Furthermore, on the day of the experiment, participants had the opportunity to practice for 5 to $10 \mathrm{~min}$ in the laboratory to familiarize themselves with the exercises.

\subsection{Wireless surface EMG}

Muscle activity was measured during the exercise task using surface EMG (Ultium EMG, EM-U810M8, Noraxon USA Inc., Scottsdale, AZ, USA) and recorded at $2000 \mathrm{~Hz}$ with band-pass filtering (10-500 Hz) on a personal computer (EM-P5, Noraxon) using a receiver (EM-U880, Noraxon). Prior to attaching the electrodes, the skin was shaved, abraded, and cleaned with alcohol. The electrode application site for EMG was determined according to previous studies $[15,16]$ and guidelines by SENIAM (URL: http://www.seniam.org/). Surface electrodes (Ambu, Blue Sensor M-00-S, Ballerup, Denmark) were attached $35 \mathrm{~mm}$ apart to the upper trapezius (UT), the lower trapezius (LT), the serratus anterior (S Ant), the latissimus dorsi (Lat), the clavicular part of the pectoralis major (PM), the external oblique (EO), the internal oblique (IO), and the rectus abdominal (RA) muscles on the right side. The electrodes for each muscle were attached precisely parallel to the muscle fibers (Table 3, Fig. 2). The skin impedance was confirmed to be less than $5 \mathrm{k} \Omega$ before each measurement [17]. All data were rectified and smoothed using a root-meansquare algorithm and with a 50-ms time reference. This experimental test was not set for the comparison of muscle activity levels between muscles. Instead, an amplitude comparison was conducted between the signals from a given muscle between the two exercise tasks performed by an individual in the same session, strictly under the same experimental conditions, and without 

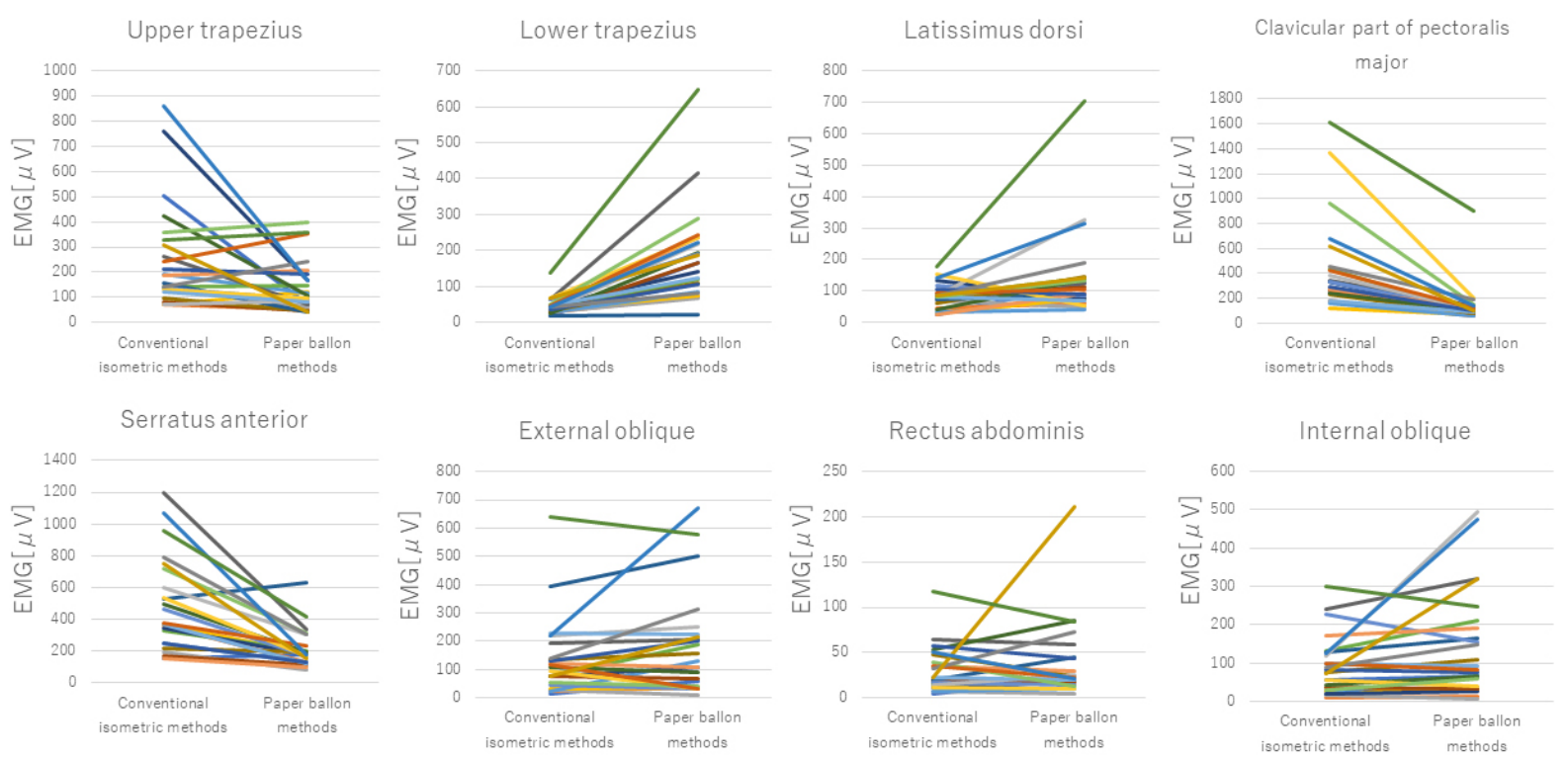

Fig. 3. Differences in muscle activity between the exercise tasks for each subject in each muscle.

altering the EMG electrodes [18,19]. The average value used for analysis $(\mu \mathrm{V}$-s) was calculated and then averaged over the 5-s isometric contraction $(\mu \mathrm{V})$. To analyze the differences in muscle activity between exercise tasks, the following formula was used to calculate a ratio for each muscle: $\mathrm{PBM} / \mathrm{CIM}$ ratio $(\%)=$ (muscle activity during $\mathrm{PBM}) /($ muscle activity during CIM) $\times 100$. A ratio above $100 \%$ indicated that the activity during the PBM was higher than that during CIM. On the other hand, a ratio below $100 \%$ indicated that the activity during CIM was higher than that during PBM.

\subsection{Statistical analyses}

Statistical analyses were performed using IBM SPSS statistics version 25.0 (IBM Corp., Armonk, NY, USA). The Shapiro-Wilk test was performed to confirm normality. Since normality could not be confirmed, the Wilcoxon signed-rank test was used to assess the difference between the exercise tasks. A $p$-value $<0.05$ was considered statistically significant in an a priori power analysis. The data are expressed as medians (interquartile ranges).

\section{Results}

The medians and interquartile ranges of muscle activity during each exercise task are shown in Table 4 . The activation of the LT $(p<0.001)$ and Lat $(p=0.030)$ were significantly higher in the PBM than in the CIM.
Table 4

Comparison of the muscle activity between the isometric squeeze exercises using the paper balloon and the hard plastic-cylinder

\begin{tabular}{lccr}
\hline \multicolumn{1}{c}{ Muscle } & $\begin{array}{c}\text { Paper } \\
\text { balloon } \\
(\mu \mathrm{V})\end{array}$ & $\begin{array}{c}\text { Hard plastic } \\
\text { cylinder }(\mu \mathrm{V})\end{array}$ & $p$-value \\
\hline Upper trapezius & $102.9(105.5)$ & $172.3(185.1)$ & 0.016 \\
Lower trapezius & $167.3(125.5)$ & $40.3(18.0)$ & $<0.010$ \\
Serratus anterior & $179.8(119.5)$ & $371.5(179.8)$ & $<0.010$ \\
Latissimus dorsi & $108.8(64.6)$ & $82.5(39.3)$ & 0.030 \\
Pectoralis major & $96.7(46.7)$ & $333.5(194.1)$ & $<0.010$ \\
External oblique & $118.8(180.5)$ & $99.4(99.2)$ & 0.496 \\
Internal oblique & $87.0(143.8)$ & $77.9(92.9)$ & 0.293 \\
Rectus abdominal & $22.1(25.8)$ & $20.9(26.7)$ & 0.592 \\
\hline
\end{tabular}

Values are medians (interquartile ranges).

The activation of the UT ( $p=0.016)$, PM $(p<0.001)$, and S Ant $(p<0.001)$ were significantly higher in the CIM than in the PBM. Both the PBM and the CIM showed activation of the trunk muscles but no significant differences in the activity of the RA $(p=0.592)$, EO $(p=0.496)$, and IO $(p=0.293)$ was observed among the exercise tasks. In Fig. 3, the muscle activity of each subject during the exercise task is shown. The bar graph in Fig. 4 expresses the ratio between exercise tasks for each muscle. The PBM/CIM ratios of the LT and LAT were $410 \%$ and $175 \%$, respectively. The PBM/CIM ratios of the UT, PM, and S Ant were 56\%, $31 \%$, and $44 \%$, respectively.

\section{Discussion}

This study investigated muscle activation during two 


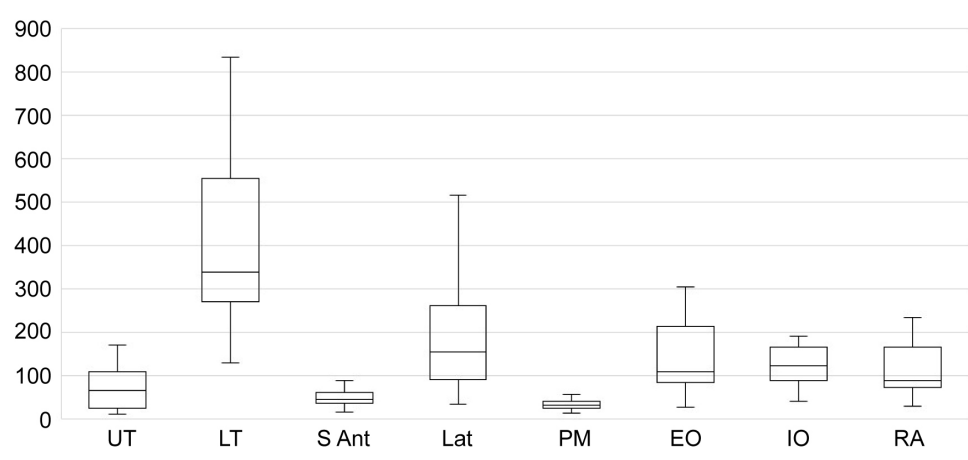

Fig. 4. The PBM/CIM ratio in each muscle. PBM, paper balloon method; CIM, conventional isometric method.

types of isometric chest squeeze exercises in the same posture and position, using external-focus instructions while holding different objects. In the conventional isometric method, participants pushed as hard as they could against the hard material, and a great amount of pressure was exerted. In the paper balloon method, the force was exerted with control to avoid crushing the paper, with the aim of preserving the shape of the object while maintaining the maximum force towards the paper balloon. The results showed that muscle activation differed between the two exercises.

The activities of the lower trapezius and latissimus dorsi were significantly higher in the paper balloon method than in the conventional isometric method. However, the activities of the upper trapezius, pectoralis major, and serratus anterior were significantly lower in the paper balloon method than in the conventional isometric method. The difference in instructions may have altered the degree of co-contraction of the muscles around the scapula. We assumed that the participants strongly activated their latissimus dorsi as the antagonistic muscle of the pectoralis major to avoid crushing the paper balloon during the paper balloon method compared to the conventional isometric method. The participants volitionally produced the maximum force toward the object while exerting control to avoid crushing the paper balloon, presumably stabilizing the scapula by activating the lower trapezius instead of utilizing the serratus anterior. Several studies have shown that the LT muscle is activated in the raised shoulder position [20,21]. For this reason, training of the lower trapezius is generally performed by raising the upper limbs. However, we set the participant's shoulder position at $45^{\circ}$ abduction, without elevating the arms. The LT muscle activity was increased by the instruction of exerting control to avoid crushing the object while maintaining the body position during an isometric exercise. In this study, the paper balloon method significantly activated the lower trapezius muscle without involving any complicated movement; moreover, significantly lower activity was maintained in the pectoralis major muscle even during maximum effort. In this respect, the present study presented new data.

The lower trapezius muscle plays an important role in stabilizing the upper body, rotator cuff, and scapular movement. Athletes with impingement symptoms show a significant increase in the upper trapezius activity but low activation of the lower trapezius during isokinetic abduction and external rotation [22]. The lower trapezius muscle-focused exercises are significantly more important for overhead sports [23]. The lower trapezius muscle strengthening exercise program is a clinically effective method that can reduce the level of neck pain or cervical dysfunction [24]. The results of this study indicate that exercises using the paper balloon method activate the lower trapezius and latissimus dorsi and significantly reduce activation of the upper trapezius, pectoralis major, and serratus anterior in the absence of instructions to change the body position or focus on specific body areas or muscle groups. Therefore, the paper balloon method, an isometric exercise method performed with control exerted to avoid crushing an object, represents a candidate exercise for restoring scapular muscle balance in shoulder rehabilitation. Supporting information on the differences between the two types of isometric exercises is shown in supplemental data.

Performing a new isometric exercise method while exerting control, so an object, such as a paper balloon, does not crush enhances the activity of the lower trapezius muscle, which may contribute to improving sports performance and preventing sports injuries. From a clinical and rehabilitation standpoint, the paper balloon method has the potential to be used in shoulder stability training for patients with physical restrictions, such as individuals with limited arm mobility, or in exercise programs for elderly people. 
This study has several limitations. First, we only assessed the participants in a single position while they applied maximum effort. Different positions can lead to different outcomes for muscle activation. Second, we only tested male participants; thus, whether women would show similar results is unknown. Lastly, we did not normalize the EMG signals since data were collected/compared in the same subject during the same session within a short time period [25]. Therefore, these should be reflected and analyzed in future studies.

\section{Conclusions}

In apparently healthy subjects who are tested at same body position, using volitional control to avoid crushing an object, the novel isometric method described herewith, enabled activation of the lower trapezius and latissimus dorsi while significantly lowering the activation of the upper trapezius, pectoralis major, and serratus anterior muscles in the absence of instructions to focus on a specific body part or muscle group. Therefore, it may contribute to improving sports performance, in preventing injuries and in rehabilitation following musculoskeletal injuries.

\section{Acknowledgments}

We would like to thank Editage (www.editage.jp) for English language editing.

\section{Author contributions}

CONCEPTION: Koji Murofushi.

PERFORMANCE OF WORK: Koji Murofushi, Kenji Hirohata and Daisuke Yamaguchi.

INTERPRETATION OR ANALYSIS OF DATA: Hidetaka Furuya, Kenji Hirohata and Sho Mitomo.

PREPARATION OF THE MANUSCRIPT: Koji Murofushi, Daisuke Yamaguchi, Kenji Hirohata and Sho Mitomo.

REVISION FOR IMPORTANT INTELLECTUAL CONTENT: Koji Murofushi, Kenji Hirohata and Sho Mitomo.

SUPERVISION: Koji Murofushi, Daisuke Yamaguchi and Kenji Hirohata.

\section{Ethical considerations}

All participants provided written informed consent for participation in this study before engaging in the trials. This study was approved by the Research Ethics Committee (approval number: M2019-295) of the participating institution and followed the principles of the Declaration of Helsinki (52nd WMA General Assembly Edinburgh, Scotland, October 2000) for Medical Research Involving Human Subjects.

\section{Conflict of interest}

The authors certify that there is no conflict of interest with any financial organization regarding the material discussed in the manuscript.

\section{Funding}

This study did not receive funding.

\section{References}

[1] Snyder BJ, Fry WR. Effect of verbal instruction on muscle activity during the bench press exercise. J Strength Cond Res. 2012; 26(9): 2394-2400. doi: 10.1519/JSC.0b013e31823f8d 11.

[2] Paoli A, Mancin L, Saoncella M, Grigoletto D, Pacelli FQ Zamparo P, et al. Mind-muscle connection: Effects of verbal instructions on muscle activity during bench press exercise. Eur J Transl Myol. 2019; 29(2): 8250. doi: 10.4081/ejtm.2019. 8250 .

[3] Sahaly R, Vandewalle H, Driss T, Monod H. Surface electromyograms of agonist and antagonist muscles during force development of maximal isometric exercises-effects of instruction. Eur J Appl Physiol. 2003; 89(1): 79-84. doi: 10.1007/ s00421-002-0762-6.

[4] Halperin I, Hughes S, Panchuk D, Abbiss C, Chapman DW. The effects of either a mirror, internal or external focus instructions on single and multi-joint tasks. PLoS One. 2016; 11(11): e0166799. doi: 10.1371/journal.pone.0166799.

[5] Porter JM, Anton PM, Wikoff NM, Ostrowski JB. Instructing skilled athletes to focus their attention externally at greater distances enhances jumping performance. J Strength Cond Res. 2013; 27(8): 2073-2078. doi: 10.1519/JSC.0b013e31827e15 21.

[6] Wulf G, Dufek JS, Lozano L, Pettigrew C. Increased jump height and reduced EMG activity with an external focus. Hum Mov Sci. 2010; 29(3): 440-448. doi: 10.1016/j.humov.2009. 11.008.

[7] Loaiza-Betancur AF, Pérez Bedoya E, Montoya Dávila J, Chulvi-Medrano I. Effect of isometric resistance training on blood pressure values in a group of normotensive participants: A systematic review and meta-analysis. Sports Health. 2020; 12(3): 256-262. doi: 10.1177/1941738120908070.

[8] McGlynn GH. A re-evaluation of isometric strength training. J Sports Med Phys Fitness. 1972; 12(7): 258-260.

[9] Oranchuk DJ, Storey AG, Nelson AR, Cronin JB. Isometric 
training and long-term adaptations: Effects of muscle length, intensity, and intent: A systematic review. Scand J Med Sci Sports. 2019; 29(4): 484-503. doi: 10.1111/sms.13375.

[10] Lum D, Barbosa TM. Brief review: Effects of isometric strength training on strength and dynamic performance. Int $\mathbf{J}$ Sports Med. 2019; 40(6): 363-375. doi: 10.1055/a-0863-4539.

[11] Vance J, Wulf G, Töllner T, McNevin N, Mercer J. EMG activity as a function of the performer's focus of attention. J Mot Behav. 2004; 36(4): 450-459. doi: 10.3200/JMBR.36.4.450459.

[12] Marchant DC, Greig M, Scott C. Attentional focusing instructions influence force production and muscular activity during isokinetic elbow flexions. J Strength Cond Res. 2009; 23(8): 2358-2366. doi: 10.1519/JSC.0b013e3181b8d1e5.

[13] Fukumori KI. The self-inflating Japanese paper balloon. Phys Today. 2017; 70(1): 78-9. doi: 10.1063/PT.3.3437.

[14] Aguilera-Castells J, Buscà B, Morales J, Solana-Tramunt M, Fort-Vanmeerhaeghe A, Rey-Abella F, et al. Muscle activity of Bulgarian squat. Effects of additional vibration, suspension and unstable surface. PLoS One. 2019; 14: e0221710. doi: 10. 1371/journal.pone.0221710.

[15] Ekstrom RA, Donatelli RA, Carp KC. Electromyographic analysis of core trunk, hip, and thigh muscles during 9 rehabilitation exercises. J Orthop Sports Phys Ther. 2007; 37(12): 754-62. doi: 10.2519/jospt.2007.2471.

[16] Ekstrom RA, Donatelli RA, Gary L, Soderberg GL. Surface electromyographic analysis of exercises for the trapezius and serratus anterior muscles. J Orthop Sports Phys Ther. 2003; 33(5): 247-58. doi: 10.2519/jospt.2003.33.5.247.

[17] Cram JR, Kasman GS. Introduction to surface electromyography. Gaithersburg, MD: Aspen Publishers Inc.; 1998.

[18] Ekstrom RA, Bifulco KM, Lopau CJ, Andersen CF, Gough JR. Comparing the function of the upper and lower parts of the serratus anterior muscle using surface electromyography. J Orthop Sports Phys Ther. 2004; 34(5): 235-243. doi: 10 2519/jospt.2004.34.5.235.

[19] McCurdy K, O’Kelley E, Kutz M, Langford G, Ernest J, Torres M. Comparison of lower extremity EMG between the 2-leg squat and modified single-leg squat in female athletes. J Sport Rehabil. 2010; 19(1): 57-70. doi: 10.1123/jsr.19.1.57.

[20] Arlotta M, Lovasco G, McLean L. Selective recruitment of the lower fibers of the trapezius muscle. J Electromyogr Kinesiol. 2011; 21(3): 403-410. doi: 10.1016/j.jelekin.2010.11.006.

[21] Park SY, Yoo WG. Selective activation of the latissimus dorsi and the inferior fibers of trapezius at various shoulder angles during isometric pull-down exertion. J Electromyogr Kinesiol. 2013; 23(6): 1350-5.

[22] Cools AM, Declercq GA, Cambier DC, Mahieu NN, Witvrouw EE. Trapezius activity and intramuscular balance during isokinetic exercise in overhead athletes with impingement symptoms. Scand J Med Sci Sports. 2007; 17(1): 25-33. doi 10.1111/j.1600-0838.2006.00570.x.

[23] Gillet B, Rogowski I, Monga-Dubreuil E, Begon M. Lower trapezius weakness and shoulder complex biomechanics during the tennis serve. Med Sci Sports Exerc. 2019; 51(12): 25312539. doi: 10.1249/MSS.0000000000002079.

[24] Park SH, Lee MM. Effects of lower trapezius strengthening exercises on pain, dysfunction, posture alignment, muscle thickness and contraction rate in patients with neck pain; randomized controlled trial. Med Sci Monit. 2020; 26: e920208. doi: 10.12659/MSM.920208.

[25] Halaki M, Ginn AK. Normalization of EMG signals: To normalize or not to normalize and what to normalize to? In: Naik GR, Ed. Computational intelligence in electromyography analysis - A perspective on current applications and future challenges. Rijeka: InTech Open; 2012. p. 175. doi: 10.5772/49957.

\section{Supplemental data: Muscle activation of typical single case between the two types of isometric exercises}
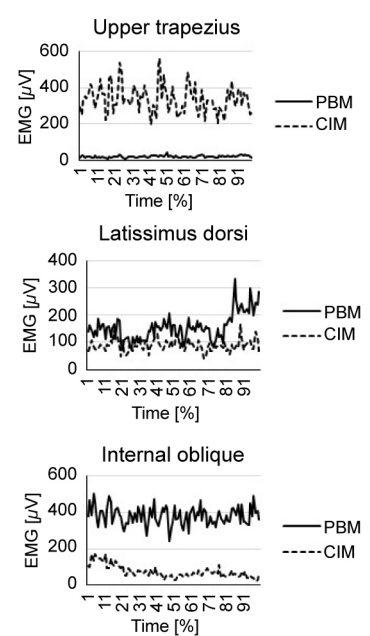

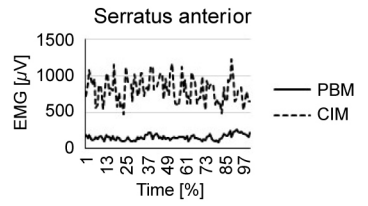

Clavicular part of pectoralis major

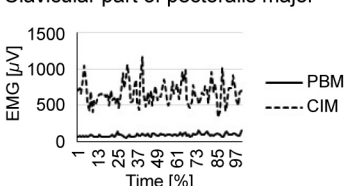

Rectus abdomis

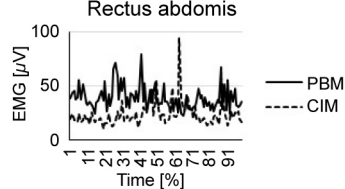

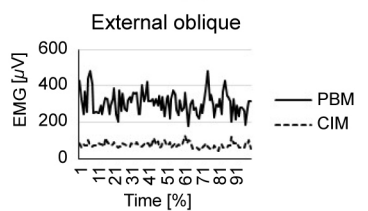

